\title{
Cahiers balkaniques
}

48 | 2021

L'évolution de la broderie de tradition byzantine en Méditerranée orientale et dans le monde slave (1200-1800)

\section{Quelques réflexions à propos de l'étoffe luxueuse} du despote de Thessalonique Andronic Paléologue, jadis conservée au monastère athonite de Vatopédi

Some thoughts on the luxurious textile of the Despot of Thessaloniki, Andronikos Palaiologos, previously conserved in the athonite Monastery of Vatopedi

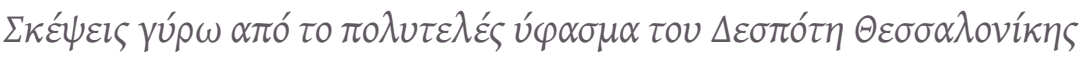

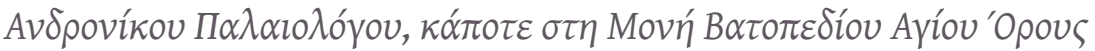

\section{Paschalis Androudis}

\section{OpenEdition}

\section{Journals}

Édition électronique

URL : https://journals.openedition.org/ceb/18405

DOI : $10.4000 /$ ceb. 18405

ISSN : 2261-4184

\section{Éditeur}

INALCO

Édition imprimée

ISBN : 9782858313983

ISSN : 0290-7402

\section{Référence électronique}

Paschalis Androudis, «Quelques réflexions à propos de l'étoffe luxueuse du despote de Thessalonique Andronic Paléologue, jadis conservée au monastère athonite de Vatopédi », Cahiers balkaniques [En ligne], 48 | 2021, mis en ligne le 24 décembre 2021, consulté le 04 janvier 2022. URL : http:// journals.openedition.org/ceb/18405; DOI : https://doi.org/10.4000/ceb.18405

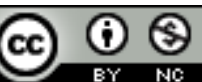

Cahiers balkaniques est mis à disposition selon les termes de la Licence Creative Commons Attribution - Pas d'Utilisation Commerciale 4.0 International. 
Quelques réflexions à propos de l'étoffe luxueuse du despote de Thessalonique Andronic Paléologue, jadis conservée au monastère athonite de Vatopédi

Résumé : le Despote de Thessalonique Andronic Paléologue (1408-1423) fut un grand donateur des monastères du Mont Athos et surtout de Vatopédi, où l'on trouve encore plusieurs de ses dons. Parmi les objets qui lui appartenaient fut une précieuse étoffe (《tapis» ou « voile»?) avec ses monogrammes. La pièce, brodée de fils d'or, est de nos jours perdue. Malgré cette perte, des dessins de l'étoffe de Fyodor Ivanovich Uspensky et de la Mission Sevastianov, ainsi que ses descriptions non détaillées, nous permettent de reconstruire son aspect réel. Dans notre article on a essayé, tant de restituer l'étoffe perdue, que de présenter son iconographie particulière, avec des motifs de caractère héraldique (aigle bicéphale, lions rampants) qui y étaient figurés.

Mots-clés : broderie au fil d'or, Vatopédi, $\mathrm{XV}^{\mathrm{e}}$ siècle

Some thoughts on the luxurious textile of the Despot of Thessaloniki, Andronikos Palaiologos, previously conserved in the athonite Monastery of Vatopedi

Abstract: The Despot of Thessaloniki Andronikos Palaiologos (1408-1423) was a great benefactor of the monasteries of Mount Athos and especially of Vatopedi, where we can still find many of his donations. Among the objects that belonged to him was a now lost precious golden-embroidered textile with its monograms. Despite this loss, designs of the textile by Fyodor Ivanovich Uspensky and the Mission Sevastianov, as well as its non-detailed descriptions, permit us to reconstruct its real aspect. In this paper I tried to reconstruct the textile and also to present its particular iconography with heraldic motifs (double-headed eagle, rampant lions).

Keywords: golden embroidered textiles, Vatopedi monastery, $15^{\text {th }}$ century 


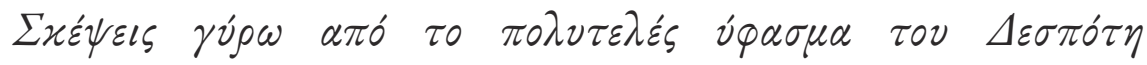

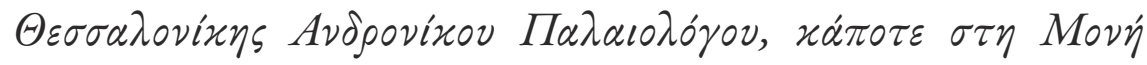

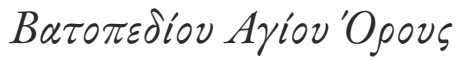

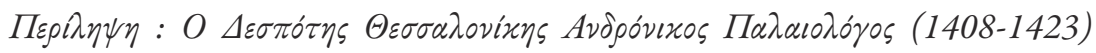

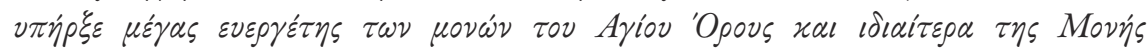

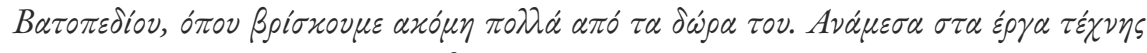

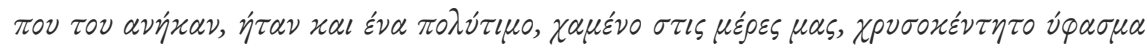

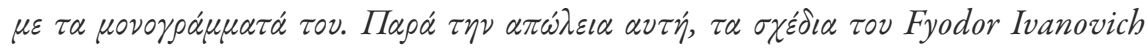

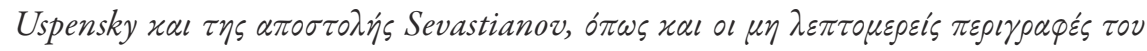

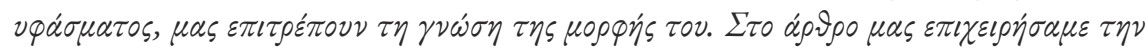

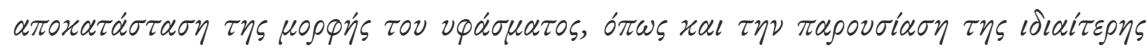

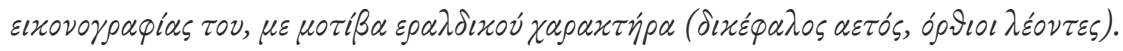

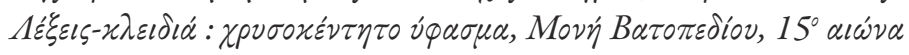




\title{
Quelques réflexions à propos de l'étoffe luxueuse du despote de Thessalonique Andronic Paléologue, jadis conservée au monastère athonite de Vatopédi
}

\author{
Paschalis Androudis \\ Université Aristote de Thessalonique \\ Je tiens à exprimer ma profonde gratitude à mes amis et collègues, les \\ Drs Elena Papastavrou et Nicolas Vryzidis pour leur aide précieuse \\ à l'amélioration du présent article.
}

Le despote de Thessalonique, Andronic Paléologue (Fig. 1)1 , était le troisième des fils de l'empereur Grec Manuel II Paléologue (1390-1425). Né probablement en $1400^{2}$, il fut nommé despote de Thessalonique en 1408. Après une courte période de gouvernement de la ville, Andronic, sous la pression de ses forces politiques, la vendit finalement aux Vénitiens en $1423^{3}$.

1. Voir sa figure dans la miniature en pleine page avec les ouvres de saint Denis l'Aréopagite (Constantinople, entre 1403 et 1405), conservée au Musée du Louvre (département des objets d'art, MR 41). Dans cette miniature, l'empereur et son fils aîné Jean VIII, déjà proclamé empereur, portent des habits lourds de pourpre violet, sans aigles. Au contraire, les deux fils puînés, les despotes Théodore et Andronic (figure 1 dans notre texte), portent des couronnes sans calotte $(\sigma \tau \xi \mu \mu \alpha \tau \alpha)$, des sceptres sans croix et de longs et lourds brocards en rouge écarlate, parsemés d'aigles bicéphales dorées, placées dans des médaillons également dorés. Sur cette miniature, voir DURAND, 1992, p. 463-464.

2. Sur le despote Andronic Paléologue voir Sideras, 1987, p. 3-15; Schreiner, 1970, p. 285-299; FERJANČIĆ, 1968, p. 227-235; TSARAS, 1965, p. 419-432.

3. Sur les documents et les conditions de la vente de Thessalonique aux Vénitiens voir SAthas, 1881, p. 135-137 ; TAFrali, 1919, p. 67-68 ; Mertzios, 1947, p. 35-37. Voir aussi NecipoĞLU, 2009, p. 105-113; JACOBY, 2002, p. 303-318. Sur la domination vénitienne à Thessalonique voir VACALOPOUlos, 1980, p. 31-52. 


\section{CAHIERS BALKANIQUES}

68 L'évolution de la broderie de tradition byzantine en Méditerranée orientale et dans le monde slave (1200-1800)

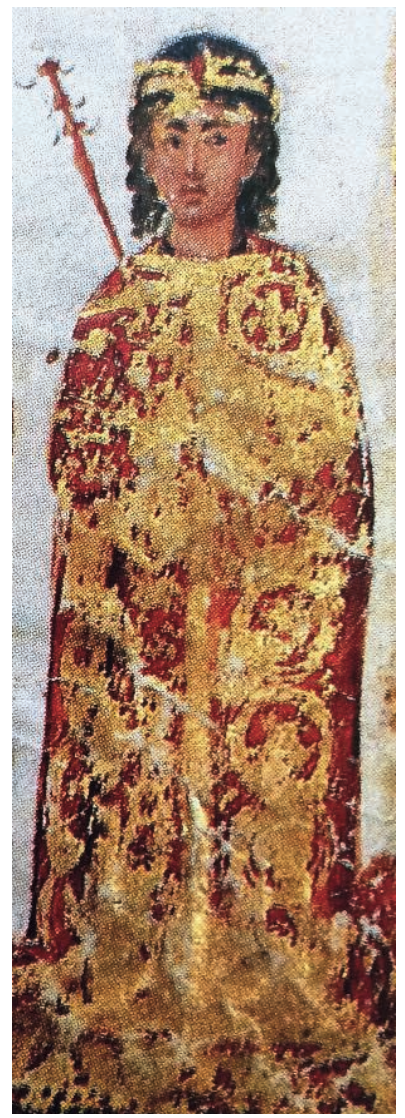

FIGURE 1. LE DESPOTE DE THESSALONIQUE ANDRONIC PALÉOLOGUE, FILS DE

L'EMPEREUR MANUEL II PALÉOLOGUE. DÉTAIL DE LA MINIATURE EN PLEINE PAGE DU MANUSCRIT AVEC LES OEUVRES SAINT DENIS L'ARÉOPAGITE (CONSTANTINOPLE, ENTRE 1403 ET 1405)

Photo: Wikimedia Commons.

Selon l'historien Apostolos Vacalopoulos, les Vénitiens, deux mois après leur entrée à Thessalonique (14 septembre 1423) ${ }^{4}$ ont arrêté Andronic et quatre autres personnages comme suspects de conspiration ${ }^{5}$. Andronic fut transporté à Nauplie (Péloponnèse) et puis se réfugia à Mantinée dans le territoire de son frère Théodore Paléologue, despote de Mistra, avant de retourner à Constantinople, où il mourra en $1429^{6}$.

Le despote Andronic Paléologue fut un grand bénéficiaire des monastères du Mont-Athos et surtout de Vatopédi ${ }^{7}$, où l'on trouve encore plusieurs de ses dons ${ }^{8}$. Parmi les objets qui lui appartenaient figurait une étoffe précieuse (« tapis » ou « voile » ?) brodée de fils d'or, dont les dimensions exactes sont inconnues, parce que l'étoffe a été malheureusement perdue. Malgré cette perte, des dessins publiés de T. Uspenskij (Fig. 2) ${ }^{9}$ et de la Mission russe de Piotr Ivanovich Sevastianov (Fig. 3) ${ }^{10}$, ainsi que de descriptions non détaillées de cette pièce, nous permettent de reconstruire son aspect. En 2001, le monastère de

4. Mertzios, 1947, p. 43.

5. Sur le départ d'Andronic et son destin voir BALFour, 1979, p. 203-205, 272-278; VaCAlopoulos, 1980, p. 36-40.

6. Voir Sideras, 1987, p. 12-14 et 1994, p. 413-418.

7. L'archéologue grec, Joachim Papangelos, a récemment émis l'hypothèse d'un possible enterrement du despote Andronic Paléologue dans le tombeau des fondateurs du monastère, situé dans le mur sud du mésonyktikon du catholicon.

8. Melvani, 2012, p. 417-429.

9. Koehne, 1871-1873, p. 1-26, pl. Lxvii. 6. Voir aussi Paliombeìs, 2003, p. 44.

10. Millet, Pargoire, Petit, 1904, no 74, pl. il. 
Vatopédi entreprit la reconstitution de l'étoffe sur la base des dessins de Sevastianov, mais sans connaître le dessin exact de l'aigle. Dans les pages suivantes on essayera, tant de restituer l'aspect réel de l'étoffe perdue, que de présenter son iconographie particulière qui est très différente de celle de la broderie religieuse de l'époque byzantine tardive ${ }^{11}$. De plus, on va donner une description des motifs de caractère héraldique (aigle bicéphale, lions rampants) qui y étaient figurés.
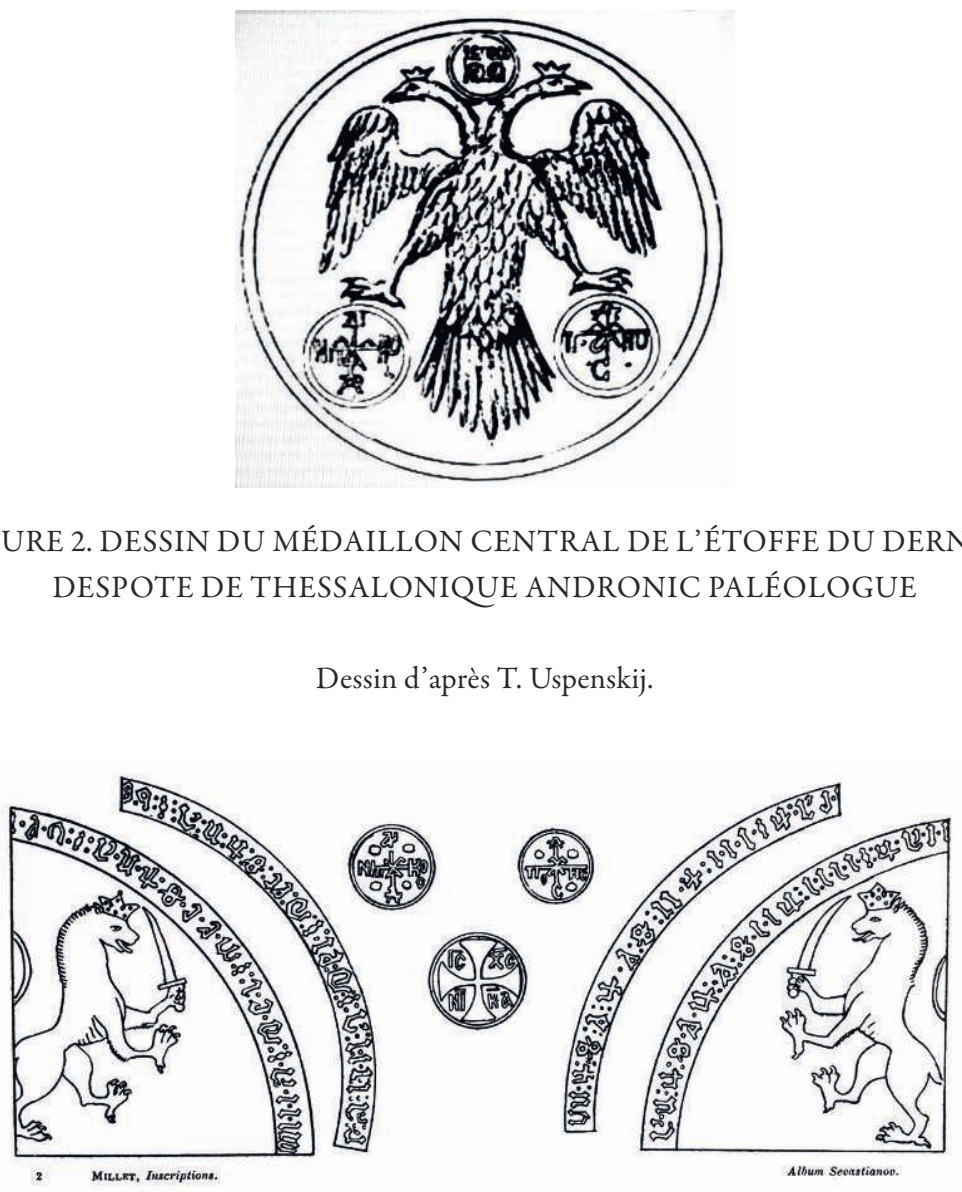

FIGURE 3. DESSIN DES CERTAINS MOTIFS DE LA MÊME ÉTOFFE

Dessin d'après Album Sevastianov

11. Sur la broderie ecclésiastique byzantine voir MiLlet, 1939 et 1947 ; WoOdfin, 2012. 


\section{CAHIERS BALKANIQUES}

70 L'évolution de la broderie de tradition byzantine en Méditerranée orientale et dans le monde slave (1200-1800)

\section{L'étoffe, description}

Malheureusement, vu la perte (ou le vol) de l'étoffe d'Andronic Paléologue, on ne peut faire de remarques ni à propos de ses dimensions qui restent inconnues, ni à propos des fils et des techniques utilisés dans son décor.

Le dessin d'Uspenskij (Fig. 2) figure donc au centre de l'étoffe un médaillon avec une aigle bicéphale ${ }^{12}$, dont chaque tête portait une petite couronne à trois pointes ; entre les têtes de l'aigle existait un petit médaillon qui enfermait une

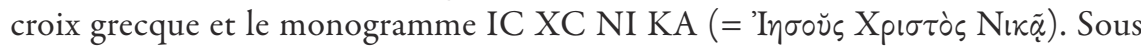
les pattes de l'aigle étaient brodés deux autres médaillons, plus grands, contenant les monogrammes qui composaient respectivement les mots AN $\triangle \mathrm{PONIKOC}$ $\Pi А \Lambda \mathrm{AIO} \Lambda \mathrm{O} Г О C$ et $\triangle \mathrm{ECПОТНС.} \mathrm{D'autre} \mathrm{part} \mathrm{le} \mathrm{dessin} \mathrm{fait} \mathrm{par} \mathrm{la} \mathrm{mission}$ Sevastianov et publié par Gabriel Millet, Jules Pargoire et Louis Petit en 1904 figure des lions rampants couronnés et tenant des épées nues, ainsi que les lettres « pseudo-latines » (?) brodées dans les registres en forme de quart de cercle placés aux écoinçons de l'étoffe ${ }^{13}$.

Outre ces témoignages graphiques, le hiéromoine Gerasimos Smyrnakès a laissé une description de l'étoffe brève et sommaire :

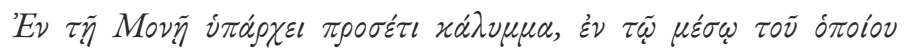

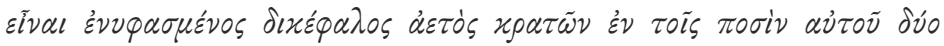

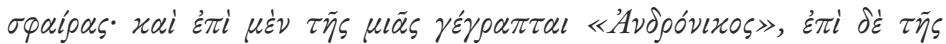

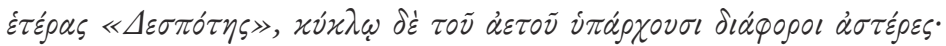

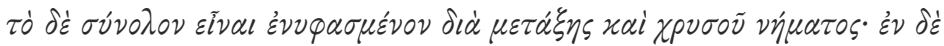

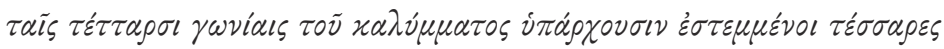

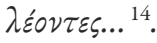

Comme on l'a vu, Smyrnakès qualifie l'étoffe de « $\kappa \dot{\alpha} \lambda \nu \mu \mu \alpha »$, ce qui signifie qu'elle était indubitablement de dimensions petites. Malheureusement, il ne donne aucun autre renseignement, par exemple sur sa forme, carrée ou rectangulaire.

De toute façon, l'étoffe d'Andronic n'était pas un « $\pi \alpha \rho \alpha \pi \varepsilon \dot{\tau} \tau \sigma \sigma \mu \alpha »$, puisque Smyrnakès qualifie de ce terme une autre étoffe du XVIII ${ }^{\mathrm{e}}$ siècle :

12. L'aigle en héraldique est toujours du genre féminin. Voir Pastoureau, 1979.

13. Millet, Pargoire, Petit, 1904, pl. ir.

14. SMyrnakès, 1988, p. 440. 


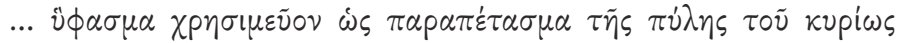

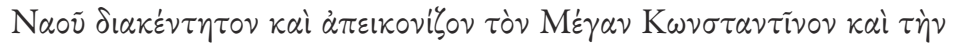

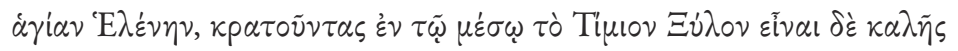

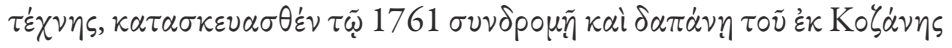

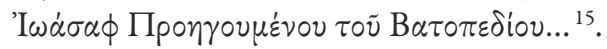

Il faut ici noter que le monastère de Vatopédi possédait une autre étoffe avec les monogrammes du despote Andronic, décrite par John Covel en 1677 comme suspendue « devant l'entrée du narthex du catholicon ${ }^{16}$. Cette étoffe, elle aussi

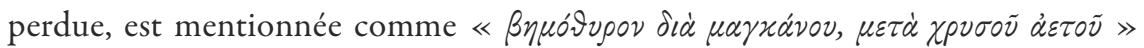
dans les proskynetaria (guides du monastère) du XviII ${ }^{\mathrm{e}}$ siècle $^{17}$. Nicolas Melvani a, à tort, distingué les deux étoffes d'Andronic comme étant deux pièces différentes, au vu des grandes différences qui présentaient leurs monogrammes ${ }^{18}$.

\section{Répertoire iconographique de l'étoffe d'Andronic}

\section{L'aigle bicéphale}

L'image de l'aigle à double tête, oiseau fabuleux, est devenue sous les Paléologues une sorte de «blason » de la famille impériale. Notons sur ce point que la sorte «d'héraldique » développée dans les confins de Byzance aux XIV ${ }^{\mathrm{e}}-\mathrm{XV}^{\mathrm{e}}$ siècles ${ }^{19}$ n'a jamais été soumise aux lois strictes du blason des pays occidentaux. L'empereur byzantin n'a, en aucun temps, octroyé d'armoiries à ses sujets. Celles-ci étaient de libre adoption, laissées aux choix du possesseur. De plus, l'ancienne emblématique familiale et locale, généralement stable quant au symbole choisi, ne s'est jamais pliée aux exigences de la loi des émaux et des métaux du blason occidental, pour ne pas parler des meubles de l'écu qui, bien des fois, présentaient un aspect des

15. Ibid., p. 440.

16. Hasluck, 1910-1911, p. 123-124.

17. Melvani, 2012, p. 421.

18. Ibid., p. 421.

19. Sur la question de l'héraldique à Byzance voir Typaldos, 1926, p. 206-222; Soloviev, 1935, p. 119-164; Cernovodeanu, 1982a, p. 339-358, 1982b, 409-422 et 1983, p. 49-54; Orкonomou, 1986, p. 9-20; SAVvidès, 1994-1995, p. 71-77; ANDroudis, 2002, p. 11-42; OUsterhout, 2009a, p. 153-170, 2009b, p. 91-109 et 2016, p. 92-97. Les anciennes opinions proposées par Hemmerdinger, 1968, p. 304-309, paraissent peu convaincantes. 


\section{CAHIERS BALKANIQUES}

72 L'évolution de la broderie de tradition byzantine en Méditerranée orientale et dans le monde slave (1200-1800)

plus insolites et hétéroclites du point de vue des normes héraldiques classiques ${ }^{20}$. Il est vrai que les Byzantins des XIII $-\mathrm{XV}^{\mathrm{e}}$ siècles n'avaient pas eu le même souci de marquer tous les objets d'art et les monuments de leurs armes et leurs noms que les Occidentaux, surtout d'ailleurs à une époque plus récente.

Selon J. Touratsoglou, dont nous partageons l'opinion :

C'est à cette époque qu'apparaissent pour la première fois l'aigle bicéphale, les grands $\mathrm{B}$, les monogrammes de la famille des Paléologues, l'empereur à cheval, l'empereur sous un arc ou la représentation de la porte des murailles de la ville ${ }^{21}$.

L'aigle bicéphale et son emploi dans la cour grecque de la période byzantine tardive ont fait couler beaucoup d'encre ${ }^{22}$. Son origine est sans aucun doute orientale ${ }^{23}$. Certains chercheurs ont considéré que le motif était arrivé dans l'Empire byzantin, soit par la filière des Turcs seldjoukides, soit par la filière occidentale, à travers les Balkans. L'aigle à double tête se trouve déjà sous le règne d'Andronic II Paléologue (1282-1328), dans les détails des portraits impériaux (le plus ancien exemplaire datant de 1301) ${ }^{24}$. En même temps, les représentations balkaniques de l'aigle bicéphale sont sensiblement plus tardives que ne l'auraient cru les chercheurs il y a quelques décennies.

On retrouve l'aigle à deux têtes, dès la fin du XIII ${ }^{\mathrm{e}}$ siècle, dans les habits des empereurs grecs et ceux des leurs fils (despotes, césars). Le traité du curopalate Georges Pseudo-Kodinos (composé entre 1347 et 1348, au début du règne de Jean vi Cantacuzène à Constantinople) ${ }^{25}$ est une source de base pour les emblèmes

20. Cernovodeanu, 1982a, p. 344.

21. Touratsoglou, 1987 , p. 185.

22. Sur l'image de l'aigle à deux têtes à Byzance voir Lambros, 1909, p. 433-473; Bees, 1912, p. 321-330; Gerola, 1934, p.7-36; Soloviev, 1935, p. 119-164; Hemmerdinger, 1968, p. 304-309 ; Spyridakis, 1972-1973, p. 162-174; Fourlas, 1980, p. 97-120 et 1984, p. 179-190 ; СнотZAKoglou, 1996, p. 60-68 et pl. I-XII.

23. Sur les plus anciennes représentations connues d'aigles chez les Turcs seldjoukides de Rûm et les princes Artuqides de Mésopotamie voir PEKER, 1989 et surtout ANDroudis, 1999, p. 309-345, avec toute la bibliographie relative.

24. Dans le détail de la miniature du Chrysobulle de Monemvasie qui représente l'empereur Andronic II Paléologue (1282-1328). De même que sur les sceaux et les monnaies le portrait impérial est considéré comme une garantie de l'authenticité et de l'efficacité du document. L'aigle y est illustrée en or, sur champ de gueules, sur les extrémités du suppedion en coussin impérial.

25. Voir Verpeaux, 1966 ; Macrides, Munitiz \& Angelov, 2013. 
de caractère héraldique portés sur le costume des dignitaires de la cour aulique de Constantinople. Ce traité nous informe que les aigles (à une ou à deux têtes) étaient très la mode dans la cour byzantine du XIv ${ }^{\mathrm{e}}$ siècle. Brodées sur les étoffes luxueuses destinées à l'usage de la cour ${ }^{26}$, sur les chaussures des princes et des despotes, sur les selles de leurs chevaux et sur leurs tentes ${ }^{27}$, elles figuraient aussi sur les selles des chevaux et les tentes des sébastocrators ${ }^{28}$. L'auteur ne mentionne nulle part que ces aigles avaient une ou deux têtes, mais on peut bien le supposer, vu que les tissus orientaux ou d'inspiration orientale présentent, dès le $\mathrm{XIII}^{\mathrm{e}}$ siècle surtout, des aigles bicéphales et pas d'aigles à une tête. Plus tard, au cours des $\mathrm{XIV}^{\mathrm{e}}$ et $\mathrm{XV}^{\mathrm{e}}$ siècles, l'aigle bicéphale apparaît plus fréquemment dans les détails des fresques (habits des souverains) ${ }^{29}$, dans les sculptures, monnaies, étoffes, et dans d'autres œuvres qui s'attachent non seulement aux membres eux-mêmes de la famille impériale, mais aussi à leurs associés par mariages.

D’après G. Sprantzès, ce sont les aigles bicéphales, brodées en perles, sur les chaussures de pourpre du dernier empereur, Constantin XI Paléologue, qui ont permis d'identifier son corps après la chute de Constantinople en 1453.

L'aigle bicéphale avec ses têtes sommées de couronnes n'apparaît que dans la deuxième moitié du XIV ${ }^{\mathrm{e}}$ siècle. Le plus beau spécimen d'une aigle bicéphale brodée se retrouve dans une pièce en soie de grandes dimensions (1,66 m x 13,9 m) conservée au Metropolitan Museum de New York et datée des années 1366-1384 environ (figures 4 et 5). Cette étoffe fut dernièrement qualifiée de broderie d'usage ecclésiastique, au lieu de bannière militaire, comme il a été proposé auparavant ${ }^{30}$.

26. Verpeaux, 1966, p. 35-40.

27. Ibid., II, p. 143.5-145.13.

28. Ibid., II, p. 147.14-148.12.

29. Plusieurs exemples dans Velmans, 1977.

30. Rogers Fund, 1912 (12. 104.1). BALL, 2004, p. 495. 


\section{CAHIERS BALKANIQUES}

74 L'évolution de la broderie de tradition byzantine en Méditerranée orientale et dans le monde slave (1200-1800)

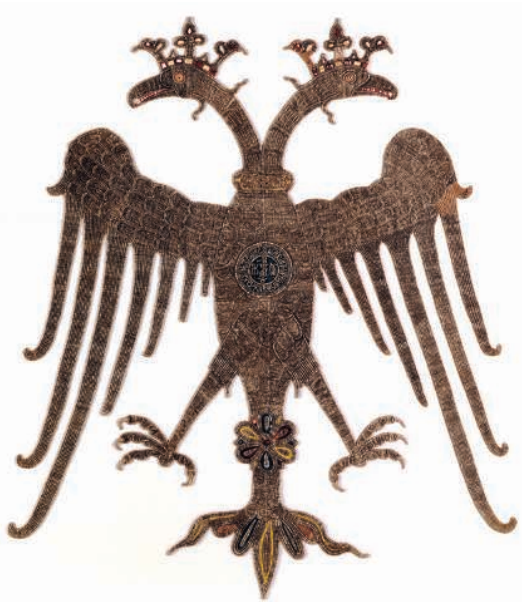

FIGURE 4. TISSU EN SOIE AVEC AIGLE BICÉPHALE BRODÉE

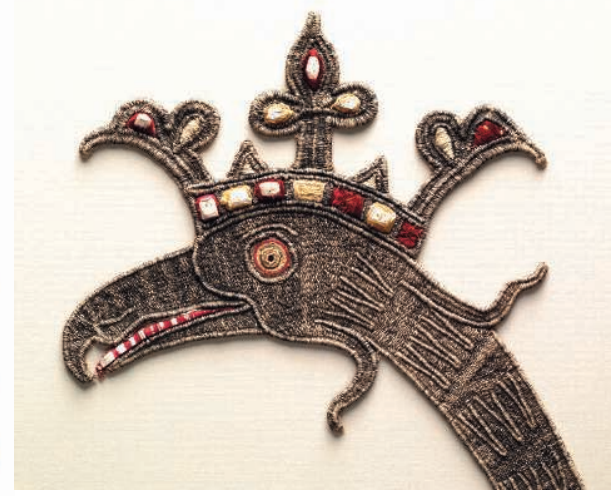

FIGURE 5. DÉTAIL DE LA FIGURE 4 : TÊTE COURONNÉE DE L’AIGLE BICÉPHALE

Photo : Metropolitan Museum, New York, Public domain.

Une autre aigle bicéphale avec les têtes sommées de couronnes, brodée de fils d'argent, figure sur les couvrures en soie bleu pâle au-dessus de deux plats en bois d'un livre contenant les œuvres de l'empereur Manuel II offert par le cardinal Bessarion au monastère de Grottaferrata, à une petite distance de Rome (Fig. 6) ${ }^{31}$.

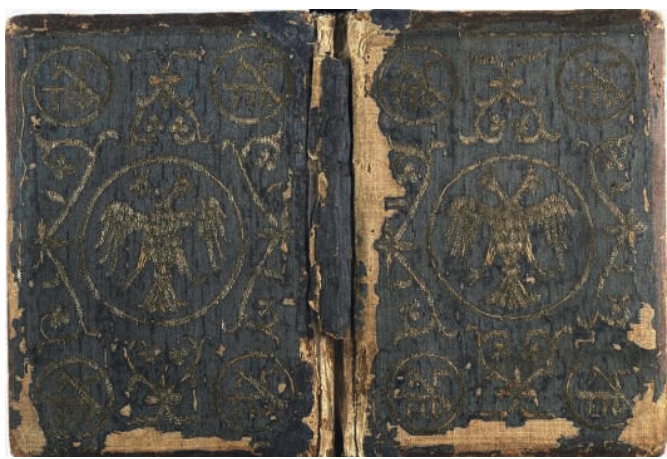

FIGURE 6. RELIURE EN SOIE (AVEC AIGLE BICÉPHALE COURONNÉE) DU LIVRE OFFERT PAR LE CARDINAL BESSARION AU MONASTÈRE DE GROTTAFERRATA

Photo : Monastère de Grottaferrata

31. Cod. Crypten. Gr. 161. Ebersolt, 1923, 111. L'oiseau fabuleux est inscrit dans un grand médaillon au centre de la couvrure, entourée de quatre médaillons portant le monogramme des Paléologues $(П А \Lambda \Gamma)$ que l'on observe aussi notamment dans des décors de manuscrits ou sur des monnaies. 
D'autres exemples d'aigles bicéphales du $\mathrm{Xv}^{\mathrm{e}}$ siècle avec leurs têtes couronnées figurent dans l'héraldique des Gattilusi, seigneurs génois de Mytilène, ancienne Phocée et Samothrace.

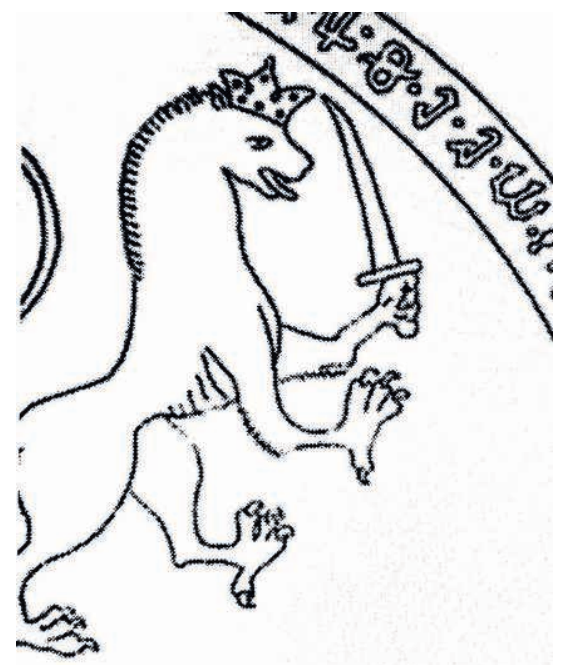

FIGURE 7. DÉTAIL DE LA FIGURE 3 : LION RAMPANT COURONNÉ

\section{Les lions rampants}

L'autre figure importante de notre étoffe byzantine est celle du lion rampant, figuré à la verticale (Fig. 7).

Le lion, représentant éminent de son espèce, était comme l'aigle un symbole solaire et lumineux à l'extrême ; roi de tous les animaux, il marquait, par excellence, la puissance royale et la force $^{32}$. Il figurait sur les habits des princes et des hauts dignitaires de l'époque médiévale.

Dans notre cas, le lion rampant est sans aucun doute une figure héraldique. On retrouve cette figure dans d'autres œuvres d'art byzantin. Citons sur ce point les lions rampants qui occupent une place centrale dans les médaillons de

la frise décorative (en champlevé) de l'église septentrionale du monastère de la Vierge Pammakaristos (Fig. 8) ${ }^{33}$.
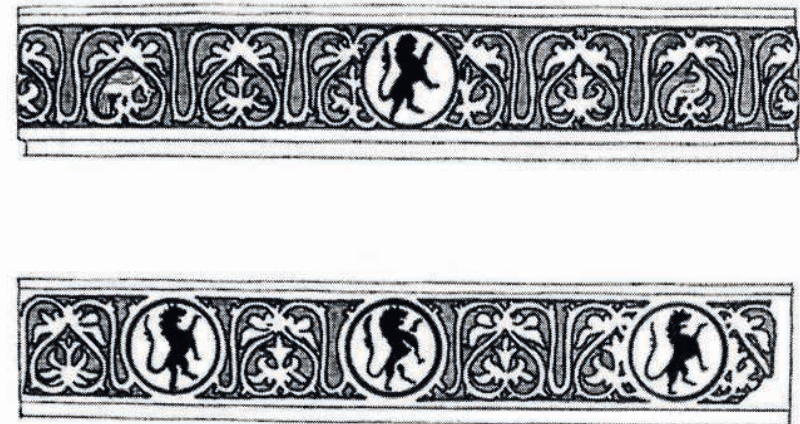

P.ANDFOUDiS
FIGURE 8. LIONS

HÉRALDIQUES

SUR LES FRISES EN

CHAMPLEVÉ DE LA

CHAPELLE SUD DU

CATHOLICON DU

MONASTÈRE DE LA

PAMMAKARISTOS À

CONSTANTINOPLE

$\left(\mathrm{XIV}^{\mathrm{E}}\right.$ SIÈCLE)

Dessin : P. Androudis

32. Sur le symbole de lion, voir Chevalier-Gheerbrant, 1982, p. 575.

33. Belting, Mango \& Mouriki, 1978, p. 21-22, Fig. 5-7, 10-11 et 94-95. 


\section{CAHIERS BALKANIQUES}

76 L'évolution de la broderie de tradition byzantine en Méditerranée orientale et dans le monde slave (1200-1800)

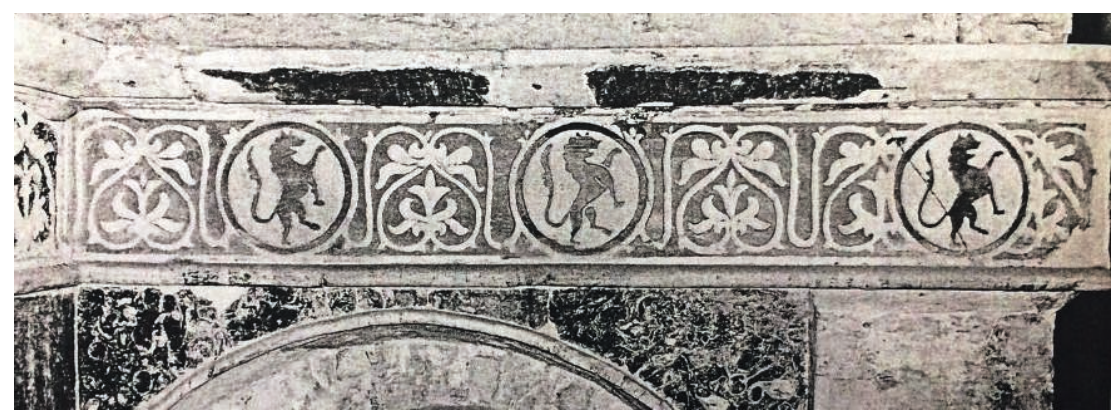

\section{FIGURE 9. DÉTAIL DE LA FIGURE 8}

De caractère incontestablement héraldique, ils figurent sur un fond rempli d'une substance colorée (mastic) en noir ou en bleu. Par contre, le contour des médaillons et les lions étaient remplis d'une matière rouge, ce qui pourrait indiquer, selon Cyril Mango, un emblème familial aristocratique byzantin. Mango signale aussi les difficultés de savoir si l'usage du lion rampant héraldique était réservé à une seule famille ou non ${ }^{34}$. Dans le cas de Pammakaristos, on devrait penser que ce lion était réservé aux fondateurs de l'église-annexe du catholicon, le prôtostratôr Michel Doukas Glabas Tarchaneiotès et sa femme Maria Doukaina Comnène Branaina Paléologue (XIV siècle $)^{35}$.

Le fragment du relief du décor sculpté de l'église-annexe du catholicon du monastère de Constantin Lips à Constantinople, déposé au musée archéologique d'Istanbul (Fig. 9), porte le lion dressé sur ses pattes de derrière. Cette église sud a eu pour fondatrice Théodora, la veuve de Michel viri Paléologue (entre 1282, date de la mort de Michel viII, et 1304, date de la mort de l'impératrice) ${ }^{36}$. Les reliefs portent d'inscriptions sépulcrales et des cadres profilés avec des figures géométriques triangulaires, polylobes et autres. Ils renferment des monogrammes des noms des ensevelis dans ces tombes, des animaux héraldiques (lions) et des rosettes ${ }^{37}$.

Un autre fragment de relief avec un lion rampant présente une allure occidentale $^{38}$. À noter la ressemblance du lion avec ceux de la dalle au-dessus de la

34. Ibid., p. 21-22.

35. Ibid.

36. Grabar, 1976, p. 127-129, pl. Civa.

37. Ibid., p. 127-129, pl. cIVa.

38. Ibid., p. 129, pl. civb. 
porte d'entrée du monastère de la Perivleptos à Mistra (deuxième moitié du XIV ${ }^{e}$ début du Xve siècle, Fig. 11) ${ }^{39}$.

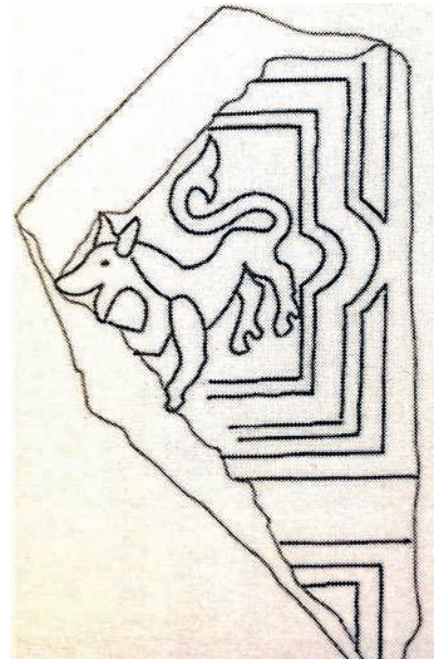

FIGURE 11. DALLE

DU MONASTÈRE

DE PERIVLEPTOS À

MISTRA

Photo : P. Androudis
FIGURE 10. FRAGMENT DE DALLE AVEC

LION HÉRALDIQUE EN BAS-RELIEF

DE L'ÉGLISE SUD DU MONASTÈRE DE

CONSTANTIN LIPS À CONSTANTINOPLE

Dessin : P. Androudis

Le lion rampant existe aussi sur d'autres sculptures constantinopolitaines, comme sur la dalle de Manuel Phakrasès Cantacuzène, en provenance de la muraille de la Corne d'Or (musée archéologique d'Istanbul, no d'inv. 4816) ${ }^{40}$.

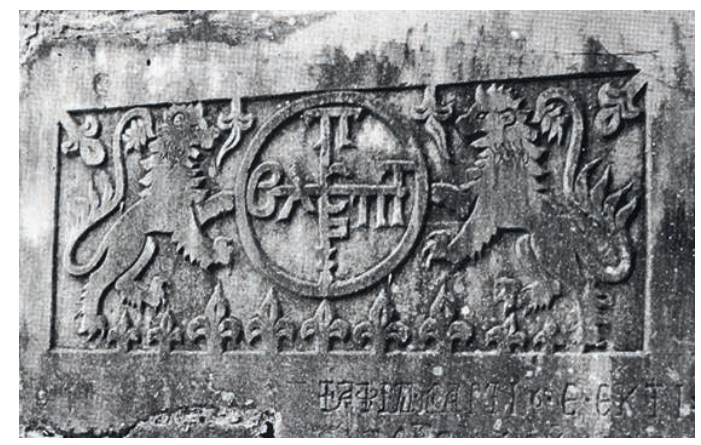

Un autre écu sculpté, autrefois encastré dans une tour de l'enceinte qui fermait l'ancien port à Kum Kapı à Constantinople, portait la figure d'un lion rampant vers la gauche, et tenant une épée. Au-dessous de l'écu, dans quatre médaillons, figuraient des monogrammes de l'empereur Andronic II Paléologue (Fig. 12) ${ }^{41}$, qui entreprit la reconstruction des fortifications de la ville ${ }^{42}$.

39. Cf. Xyngopoulos, 1953, p. 5, notes 1 et 2 ; Mouriki, 1987, p. 212, Fig. 7 et 8 ; LiVERI, 1996, p. 51, Fig. 63.

40. SCHNEIDER, 1950, p. 97.

41. Van Millingen, 1899, p. 189.

42. Gregoras, vii, p. 275 ; Nicéphore Calliste dans la dédicace de son Histoire à Andronic II. 


\section{CAHIERS BALKANIQUES}

78 L'évolution de la broderie de tradition byzantine en Méditerranée orientale et dans le monde slave (1200-1800)

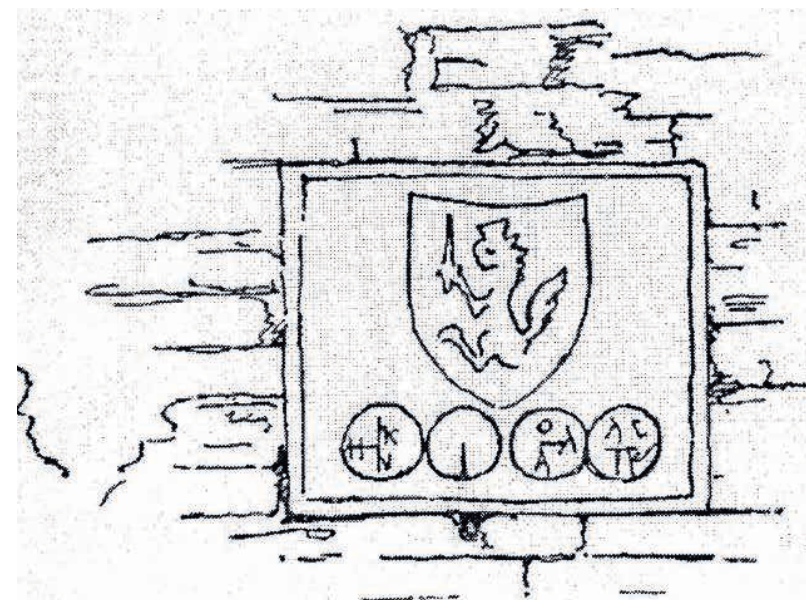

FIGURE 12. ÉCU SCULPTÉ, À CONSTANTINOPLE PORTANT UN LION RAMPANT ET LES MONOGRAMMES DE L'EMPEREUR ANDRONIC II PALÉOLOGUE

Dessin : A. Van Millingen

Ce type de lion rampant qui tient une épée est à rapprocher d'exemples analogues au-dessus de la porte d'entrée de l'enceinte du monastère de la Perivleptos à Mistra et ceux de notre étoffe luxueuse du despote Andronic Paléologue.

Rappelons aussi l'usage du lion rampant héraldique dans d'autres provinces grecques, comme à Arta (décor des plaques en champlevé de l'église de la Vierge Paregoretissa, datées entre 1283 et 1296) ${ }^{43}$, à Thessalonique (décor du couvercle prismatique du sarcophage de Georgios Kappandritis, déposé au monastère de Vlatadôn ${ }^{44}$, détail du décor d'une plaque funéraire fragmentée, jadis à la collection des sculptures de la rotonde) $)^{45}$, ainsi que dans l'église de Porta Panagia en Thessalie

43. Orlandos, 1963, p. 96-103 ; Grabar, 1976, p. 146, pl. cxxvinid.

44. Ce couvercle est sculpté en champlevé avec des losanges et des motifs floraux. Les creux du décor ont dû être remplis de mastic coloré. L'écusson du défunt qui a dû être rempli de pâte ou de mastic coloré qui ne subsiste plus, est porté par deux lions rampants ; placés de part et d'autre de l'écu. La posture des lions est incontestablement héraldique. Cf. Xyngopoulos, 1935, p. 64-78 et 1940, p. 145-148; Grabar, 1976, p. 151, pl. CXXXiII ; Pazaras, 1988, p. 35, pl. 25.

45. Sur ce fragment en champlevé voir Pazaras, 1988, p. 31, pl. 19, qui l'attribue au $\mathrm{XIV}^{\mathrm{e}}$ siècle. 
$(1283)^{46}$. Notons aussi les lions héraldiques cerclés, la tête contournée, dans le décor de la robe de Dessilava, épouse du sébastocrator Kalojan, peinte dans l'église de Boïana en Bulgarie (1259) ${ }^{47}$.

Les lions rampants apparaissent aussi dans une miniature du Roman d'Alexandre (manuscrit de Venise), illustrant la tente du roi (f. 108v). Les lions y sont peints en or, sur champ de gueules ${ }^{48}$.

Une autre variante du lion rampant est celle du lion passant, d'ailleurs figuré dans les anciennes étoffes byzantines des $\mathrm{X}^{\mathrm{e}}$-XI ${ }^{\mathrm{e}}$ siècles ${ }^{49}$; on retrouve ce type de lion sur le costume d'un personnage peint sur le tympan d'un arcosolium de l'exonarthex du catholicon du monastère de Chora. Des lions identiques figurent sur quelques bannières de guerre byzantines, tandis que les lions des étendards musulmans étaient différents (de sable sur fond de gueules) ${ }^{50}$.

Il semble que la présence du lion dans l'héraldique byzantine de la capitale soit due à une influence occidentale. On retrouve des lions héraldiques surtout à l'emblématique génoise qui s'est développée dans les territoires grecs surtout au $\mathrm{XV}^{\mathrm{e}}$ siècle. Citons à titre d'exemple la dalle de la forteresse de Mytilène, avec des lions rampant à la verticale et tenant un écu armorié (figures 12 et 13).

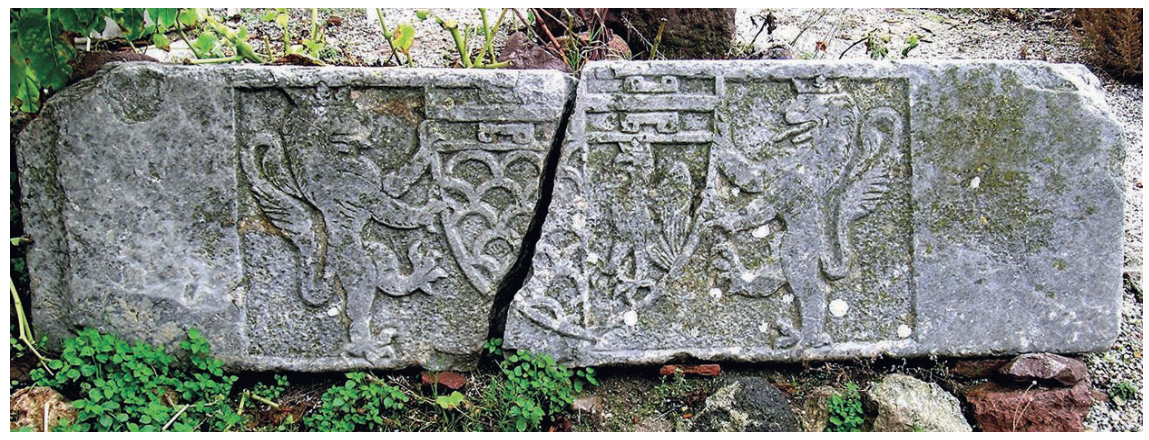

FIGURE 13. DALLE ARMORIÉE DES GATTILUSI

DANS LA FORTERESSE DE MITYLÈNE

Photo : P. Androudis

46. Grabar, 1976, p. 149, pl. cxxxic et Asimakopoulou-Atzaka, 1980, pl. 74a-b.

47. Velmans, 1977, p. 70, 96, 136, pl. xx, Fig. 54.

48. Xyngopoulos, 1965, pl. 65, 131 (f. 108v).

49. Comme par exemple dans l'étoffe de Diözesanmuseum de Cologne.

50. Citons à titre d'exemple l'étendard figuré dans deux miniatures du Roman d'Alexandre le Grand du manuscrit de l'Institut hellénique de Venise. 


\section{CAHIERS BALKANIQUES}

80 L'évolution de la broderie de tradition byzantine en Méditerranée orientale et dans le monde slave (1200-1800)

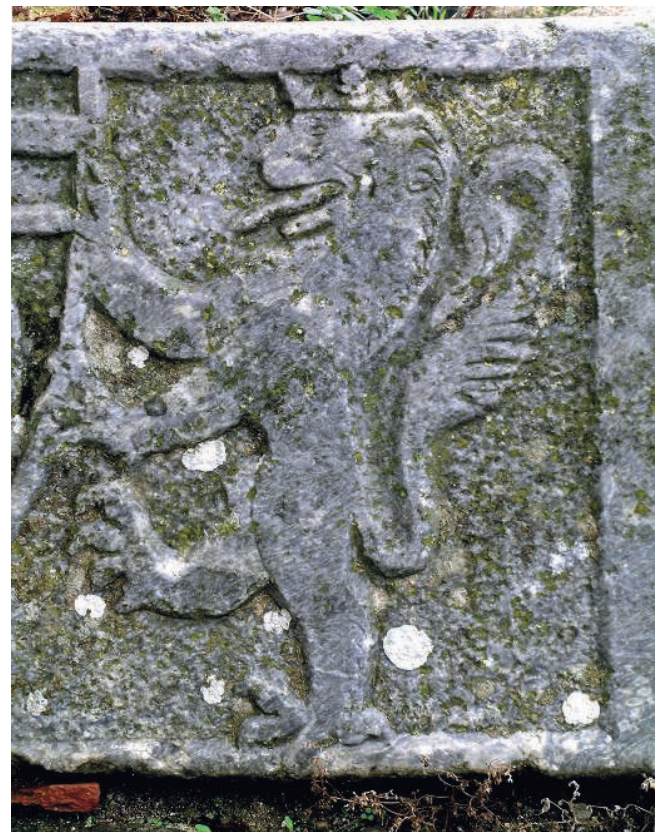

FIGURE 14. DÉTAIL DE LA FIGURE 13. LION HÉRALDIQUE COURONNÉ
La présence du lion dans l'emblématique byzantine reste, jusqu'à présent, inexpliquée. En outre, le contenu symbolique du lion n'a jamais été d'une richesse exceptionnelle. Dans les bestiaires du Moyen Âge, il évoque, d'une manière assez banale, la force, le courage, la générosité.

À noter ici que le dessin héraldique du lion de notre étoffe n'a rien de naturaliste. Il est d'ailleurs aussi probable que la plupart des artistes byzantins n'avaient jamais vu de lions véritables.

Dans les armoiries, le lion est toujours vu de profil. Sa position normale est d'être figuré verticalement sur sa patte postérieure droite, les trois autres levées, et la queue dressée parallèlement au corps. Le terme rampant est un terme qu'il est inutile d'inclure dans le blasonnement puisqu'il s'agit de son attitude ordinaire. En revanche, lorsqu'il se présente dans une position différente, il faut le préciser.

\section{Les registres « épigraphiques 》}

Quant aux registres « épigraphiques » dans les coins de l'étoffe, ceux-ci portaient des caractères très étranges et difficiles à déchiffrer (Fig. 3). G. Millet, J. Pargoire et L. Petit ont déjà qualifié ces Lettres de «pseudo-latines ${ }^{51}$. À noter que les mêmes bandes en forme de quart de cercle placées dans les écoinçons de l'étoffe se retrouvent dans d'autres broderies de l'époque Paléologue, comme les épitaphioi, mais ceux-ci ne portent ni lettres, ni autre décor.

51. Millet, Pargoire \& Petit, 1904, p. 25. 
L'usage de bandes épigraphiques orientales, pseudo-coufiques ou pseudo-latines (qui semble d'être notre cas) est une évidence assez courante dans les étoffes byzantines tardives du XIV et du Xv' siècle $^{52}$.

Il paraît, par manque de précisions et de documentation photographique, que les bandes « épigraphiques » de notre étoffe sont d'un caractère purement décoratif. D’après N. Melvani, les lettres de ces bandes étaient vraiment « gothiques », mais à l'époque où elles furent dessinées, elles étaient très abîmées ${ }^{53}$.

\section{Conclusion}

La précieuse étoffe du despote de Thessalonique, Andronic Paléologue, fut un des rares exemples de son type qui est très emblématique quant au processus de l'échange artistique et culturel marquant la période du règne des Paléologues et l'usage des motifs de caractère héraldique par la cour impériale. Il s'agit d'une œuvre d'art profane et non pas ecclésiastique.

Il est évident que les motifs de ce caractère dans les étoffes de luxe des $\mathrm{XIV}^{\mathrm{e}}-\mathrm{XV}^{\mathrm{e}}$ siècles, destinées à l'usage des membres de la famille régnante à Constantinople, ne sont pas d'éléments purement décoratifs, mais des signes figurés à valeur emblématique, de devises. Ces signes, devises, couleurs, chiffres et monogrammes qui fleurissaient chez la famille impériale et chez les hauts dignitaires de la cour aulique de Byzance, font aux armoiries traditionnelles une concurrence multiforme.

À cet égard, l'étoffe d'Andronic Paléologue déposée au monastère athonite de Vatopédi présente un grand intérêt quant à l'assimilation d'éléments emblématiques d'origine occidentale dans l'art impérial Paléologue. Ces éléments sont certes peu nombreux et ne sont pas toujours évidents, si l'on excepte les lions rampants et les fleurs de lys.

La cour de Constantinople du Xve siècle, dont le despote Andronic Paléologue faisait partie, était, comme les autres cours médiévales, une société emblématisée, caractérisée par cette sorte d'emblématique étroitement liée au paraître ${ }^{54}$. D'après les motifs héraldiques et les signes figurés de valeur emblématique figurés dans le décor de l'étoffe précieuse du despote Andronic, nous sommes donc en présence d'une codification d'éléments héraldiques, même si leur représentation ressort d'un emploi sensiblement différent de celui de l'héraldique occidentale.

52. Une très bonne recherche sur ce sujet est faite dans VRYZIDIS, 2019, p. 85-99.

53. Melvani, 2012, p. 421.

54. Androudis, 2012, p. 37. 


\section{CAHIERS BALKANIQUES}

82 L'évolution de la broderie de tradition byzantine en Méditerranée orientale et dans le monde slave (1200-1800)

\section{Bibliographie}

\section{Monographies}

Asimakopoulou-Atzaka Panagiota [Asimakoпornor-Atzaka

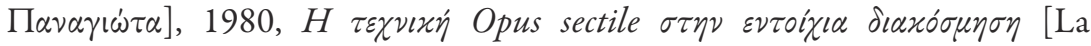
technique de l'opus sectile dans la décoration murale], Apı $\sigma \tau о \tau \dot{\lambda} \lambda \varepsilon ı$

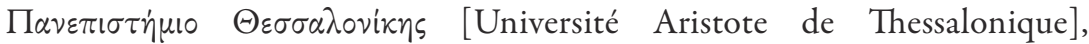
$\Theta \varepsilon \sigma \sigma \alpha \lambda$ ovikn [Thessalonique], 338 p.

BAlfour David, 1979, Politico-historical Works of Symeon Archbishop of Thessalonica (1416/17 to 1429). Critical Greek Text with Introduction and Commentary, Verlag der österreichischen Akademie der Wissenchaften, Wien, $319 \mathrm{p}$.

Belting Hans, Mango Cyril \& Mouriki Doula, 1978, The Mosaics and frescoes of St. Mary Pammakaristos (Fethiye Camii) at Istanbul, Dumbarton Oaks center for Byzantine studies, Washington, 118 p., 120 fig.

Chevalier Jean, Gheerbrant Alain, 1982, Dictionnaire des symboles, Bouquin, Paris, $850 \mathrm{p}$.

Durand Jannic (dir.), 1992, Byzance, L'Art Byzantin dans les Collections publiques Françaises (Catalogue d'Exposition d'art Byzantin au Louvre, 1992), Paris, $528 \mathrm{p}$.

EBERSOLt Jean, 1923, Les arts somptuaires de Byzance : Étude sur l'art impérial de Constantinople, Paris, $167 \mathrm{p}$.

Grabar André, 1976, Sculptures byzantines du Moyen Âge (xi ${ }^{e}$-xive siècle), A. \& J. Picard, Paris, 166 p.

Liver I Angeliki, 1996, Die byzantinischen Steinreliefs des 13. und 14. Jabrhunderts

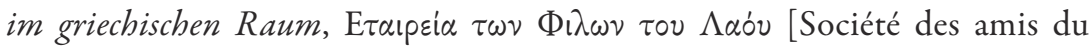

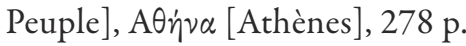

Macrides Ruth, Munitiz Joseph \& Angelov Dimiter (eds), 2013, PseudoKodinos and the Constantinopolitan Court: Offices and Ceremonies, FarnhamBurlington, UK-VT, 579 p. 


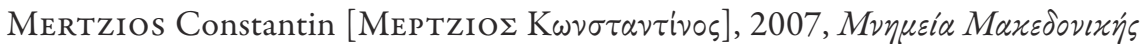

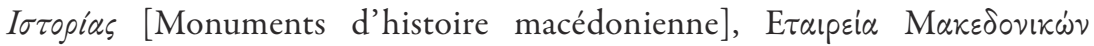
$\Sigma \pi \circ v \delta \dot{\omega} v$ [Société des études macédoniennes], $\Theta \varepsilon \sigma \sigma \alpha \lambda \circ v i \kappa \eta$ [Thessalonique], $612 \mathrm{p}$.

Millet Gabriel, Pargoire Jules \& Petit Louis, 1904, Recueil des Inscriptions chrétiennes du Mont Athos, Fontemoing, Paris, $192 \mathrm{p}$.

Millet Gabriel, 1939-1947, Broderies religieuses de style byzantin, PUF, Paris, 2 vol. 118 p. et 207 p de planches.

Millingen Van Alexander, 1899, Byzantine Constantinople. The Walls of the City, Macmillan \& co, London, 361 p.

NecipoĞLu Nevra, 2009, Byzantium between the Ottomans and the Latins, Cambridge University Press, Cambridge, 374 p.

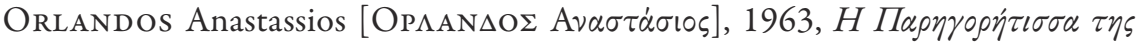

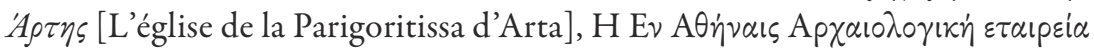
[Société Archéologique d'Athènes], AӨńva [Athènes],183 p.

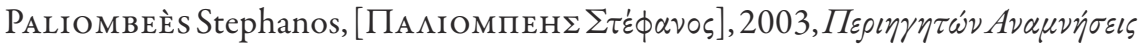

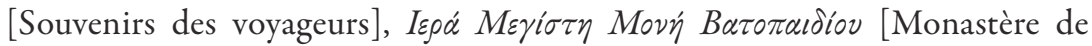
Vatopedi] Mont Athos, $319 \mathrm{p}$.

Pastoureau Michel, 1979, Traité d'Héraldique, A \& J.Picard, Paris, 406 p.

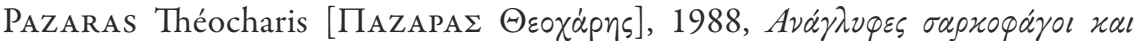

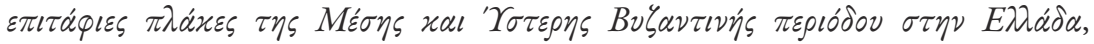

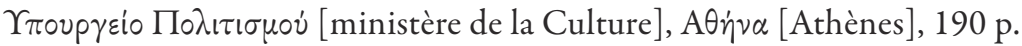

Pener Ali, 1989, The double-headed eagle of the Seljuks. A historical study, MA Thesis, Bogaziçi University, Istanbul.

Sathas C.N., 1881, Documents inédits relatifs à l'bistoire de la Grèce au Moyen Age, 1, Paris.

Sideras Alexander, 1994, Die byzantinischen Grabreden. Prosopographie, Datierung, Überlieferung, 142 Epitaphien und Monodien aus dem 


\section{CAHIERS BALKANIQUES}

84 L'évolution de la broderie de tradition byzantine en Méditerranée orientale et dans le monde slave (1200-1800)

Byzantinischen Jahrtausend, Verlag der österreichischen Akademie der Wissenchaften, Wien, $536 \mathrm{p}$.

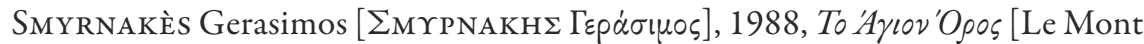

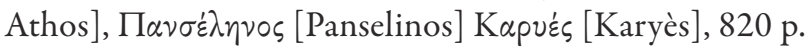

Tafrali Oreste, 1919, Thessalonique des origines au xive siècle, Paris.

Velmans Tania, 1977, La peinture murale byzantine à la fin du Moyen Age, Klincksieck, Paris, 319 p.

Verpeaux Jean (dir.), 1966, Pseudo-Kodinos, Traité des Offices, Éditions CNRS, Paris, $420 \mathrm{p}$.

Woodfin Warren, 2012, The Embodied Icon. Liturgical Vestments and Sacramental Power in Byzantium, Oxford University Press, Oxford, 374 p.

Xyngopoulos André, 1966, Les miniatures du Roman d'Alexandre le Grand dans le Codex de l'Institut Hellénique de Venise, Institut hellénique de Venise, Athènes-Venise.

\section{Articles et contributions à des ouvrages}

ANDROUdis Paschalis, 2002, « Sur quelques emblèmes héraldiques à

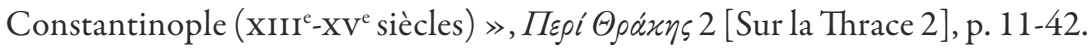

Androudis Pascal, 1999, « Origines et symbolique de l'aigle bicéphale des

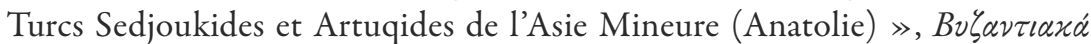
19 [Choses byzantines 19], p. 309-345, avec toute la bibliographie sur le sujet.

Ball Jennifer, 2004, "298. Podea of Paul, Patriarch of Constantinople" in Evans Helen (ed.), Byzantium. Faith and Power (1261-1557), The Metropolitan Museum of Art, N. York, Yale University Press, New Haven and London (Catalogue d'Exposition, Metropolitan Museum, 23 march-4 july 2004), New York.

Bees Nikos, 1912, „Zum Thema der Darstellung des zweiköpfigen Adlers bei den Byzantinern", Repertorium für Kunstwissenschaft 35, 1, p. 321-330. 
Cernovodeanu Dan, 1982a, « Contribution à l'étude des origines lointaines de l'héraldique (Moyen-Orient) et son développement du XII ${ }^{\mathrm{e}}$ au XV ${ }^{\mathrm{e}}$ siècle à Byzance et dans le Sud-Est européen », Genealogica et heraldica, Report of the 14th International congress, p. 339-358.

Cernovodeanu Dan, 1982b, «Contributions à l'étude de l'héraldique byzantine et post-byzantine », Actes du xvi Congrès International d'Études Byzantines, Wien 1981, Akten II/2, JÖB 32.2, p. 409-422.

Cernovodeanu Dan, 1983, « L'apparition des armoiries dans le Sud-Est européen $\gg$, Les origines des armoiries (Actes $\mathrm{du} \mathrm{II}^{\mathrm{e}}$ Colloque International d'Héraldique, Bressanone-Brixen, 5-9.X.1981), Paris, p. 49-54.

Chotzakoglou Charalambos, 1996, „Die Palaiologen und das früheste Auftreten des byzantinischen Doppeladlers", Byzantinoslavica LVII, p. 60-68 et pl. I-XII.

FerJančić Božidar, 1968, „Despot Andronik Paleolog u Solunu“ [Le despote Andronic Paléologue et Salonique], Zbornik Filozofskog Fakulteta u Beogogradu 10, p. 227-235.

Fourlas Athanasios, 1980, „Adler und Doppeladler. Materialen zum 'Adler in Byzanz'", Philoxenia: Prof. Dr. Bernhard Kötting gewidmet von seinen griechiscen Schülern Münster, p. 97-120.

Fourlas Athanasios, 1984, „Adler und Doppeladler: Kunstgeschichtliche Zeugnisse zum palaiologischen Doppeladler", Thiasos ton Mouson: Studien zu Antike und Christentum; Festschrift für Josef Fink zum 70. Geburtstag, Cologne, p. 179-190.

Gerola Giuseppe, 1934, "L'aquila bizantina e l'aquila imperiale a due teste", Felix Ravenna 43, p. 7-36.

Hasluck Frederic William, 1910-1911, "The First English Traveller's Account of Athos", The Annual of the British School at Athens 17, p. 123-124.

Hemmerdinger Bertrand, 1968, « Deux notes d'Héraldique », Byzantinische Zeitschrift 61, p. 304-309. 


\section{CAHIERS BALKANIQUES}

86 L'évolution de la broderie de tradition byzantine en Méditerranée orientale et dans le monde slave (1200-1800)

JACOBy David, 2002, « Thessalonique de la domination de Byzance à celle de Venise. Continuité, adaptation ou rupture ? », Travaux et Mémoires 14, p. 303-318.

Koenne von Berhnard, 1871-1873, „Vom Doppeladler“, Berliner Blätter für Münz, Siegel- und Wappenkunde, Vol. 6, p. 1-26, pl. LXVII.6.

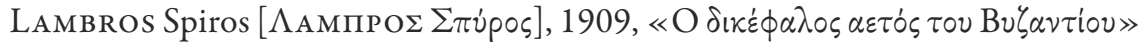

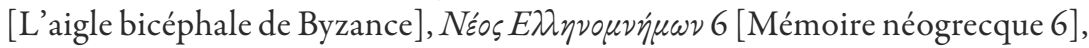
p. 433-473.

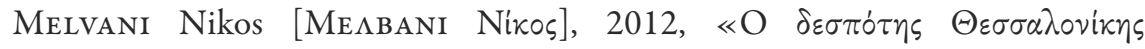

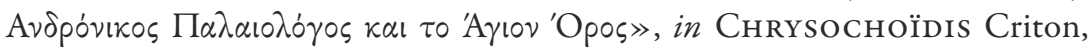
Theocharidis Ploutarchos, Papaggelos Ioakim et al.

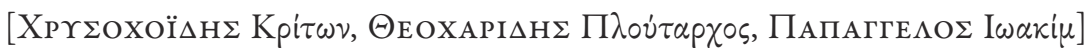

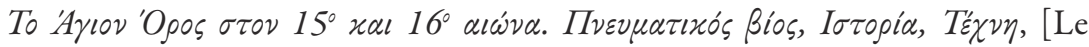
Mont Athos aux $\mathrm{Xv}^{\mathrm{e}}$ et $\mathrm{XVI}^{\mathrm{e}}$ siècles] Actes du $\mathrm{VI}^{\mathrm{e}}$ Congrès International d'Hagioreitikè Estia (Thessalonique, 25-27 novembre 2011), Athos Center, $\Theta \varepsilon \sigma \sigma \alpha \lambda \circ v_{i k \eta}$ [Thessalonique], p. 417-429.

Mouriki Doula, 1987, "Palaeologan Mistra and the West", First International Byzantine Conference (Delphi, 20-24 juillet 1985), Athènes.

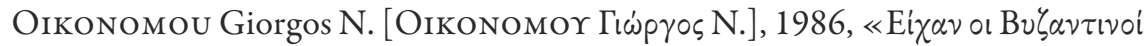

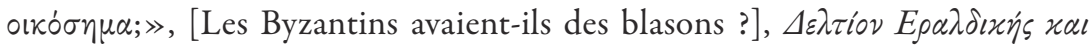

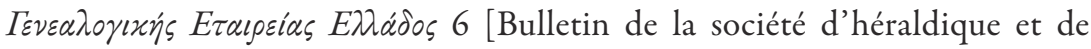
généalogie de Grèce], p. 9-20.

Ousterhout Robert, 2009a, "Byzantium between East and West and the Origins of Heraldry", in Hourinane Colum (ed.), Byzantine Art: Recent Studies, Turnhout Brepols, Antwerps, p. 153-170.

Ousterhout Robert, 2009b, „Symbole der Macht. Mittelalterliche Heraldik zwischen Ost und West" (traduction de l'anglais S. Zink), in Mersch Margit, RitZeRfeld Ülrike (eds.), Lateinisch-griechisch-arabische Begegnungen. Kulturelle Diversität im Mittelmeerraum des Spätmittelalters, Akademie, Berlin, p. 91-109. 
Ousterhout Robert, 2016, "Emblems of Power in Palaiologan Constantinople", in NecipoĞlu Nevra, Ödekan Ayla, Akyürex Engin (eds.), The Byzantine Court: Source of Power and Culture (Second International Sevgi Gönül Byzantine Studies Symposium Proceedings, Istanbul, 21-23 June 2010), Istanbul, p. 92-97.

SAVvidès Alexis, 1994-1995, "Notes on Byzantine Heraldry", Ai $\pi \tau v \chi \alpha 6$ [Diptyque 6], p. 71-77.

Schneider, Alfons Maria, 1950, „Mauern und Tore am Goldene Horn zu Konstantinopel“, Nachrichten von der Wissenschaften zu Göttingen, Philologisch-historiche Klausse 5, p. 65-107.

SChreiner Peter, 1970, „Chronologische Untersuchungen zur Familie Kaiser Manuels II", Byzantinische Zeitschrift 63, p. 285-299.

Sideras Alexander, 1987, "Neue Quellen zum Leben des Despotes Andronikos Palaiologos", Byzantinische Zeitschrift, Vol. 80/1, p. 3-15.

Soloviev Vladimir, 1935, « Les emblèmes héraldiques de Byzance et les Slaves », Seminarium Kondakovianum 7, p. 119-164.

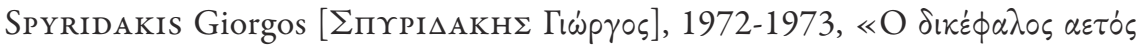

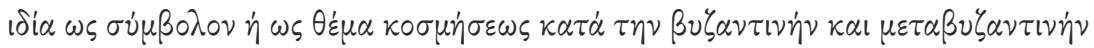

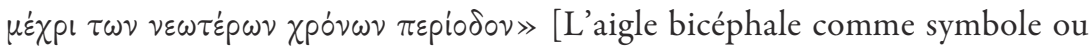
comme type de décoration pendant la période byzantine et postbyzantine

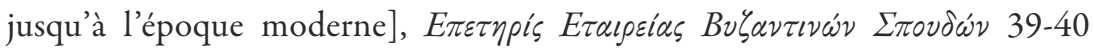
[Annuaire de la société des études byzantines 39-40], p. 162-174.

Touratsoglou Jean, 1987, « L'atelier monétaire de Thessalonique au quatorzième siècle apr. J.-C. : Le rayonnement d'un centre artistique avant le déclin de l'empire », in SAMARdzic Radovan (dir.), L'art de Thessalonique et des pays balkaniques et les courants Spirituels au xive siècle, Académie serbe des sciences et des arts, Belgrade, p. 183-193.

Tsaras Jean, 1965, « La fin d'Andronic Paléologue dernier despote de Thessalonique », Revue des Études Sud-Est Européennes 3, p. 419-432. 
Typaldos Georgios [TҮחa

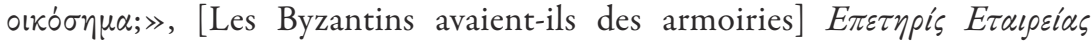
$B v \zeta \alpha v \tau \imath \nu \omega \nu \nu$ $\Sigma \pi 0 v \delta \omega_{\nu} 3$ [Annuaire de la société d'études byzantines], p. 206-222.

Vacalopoulos Apostolos [Baka

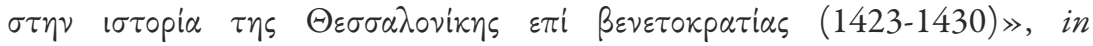

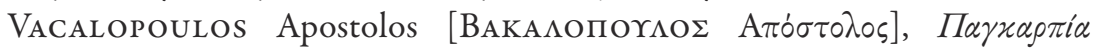

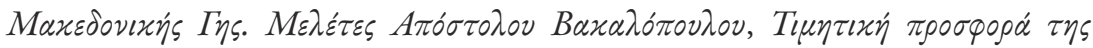

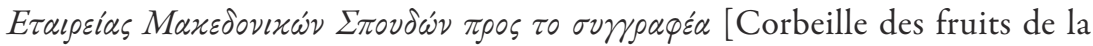
terre macédonienne, études d'Apostolos Vacalopoulos, hommage de la société

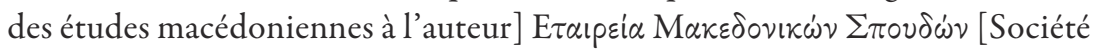

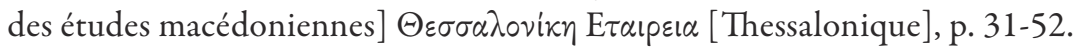

VRYZIDIs Nikolaos, 2019, “The 'Arabic' Stole of Vatopediou Monastery : Traces of Islamic Material Culture in Late Byzantium”, in NecipoĞLU Gülrü, Metzler Maria (eds.), Muqarnas 36, Leiden/Boston, p. 85-99.

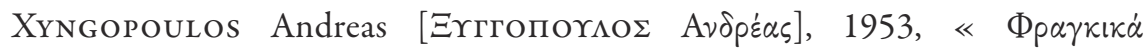

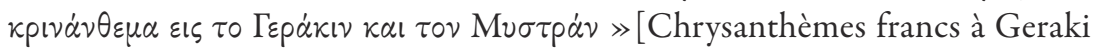
et à Mystra], Mélanges offerts à Octave et Melpo Merlier à l'occasion du 25e anniversaire de leur arrivée à Athènes, IFA, Athènes, 2, p. 205-211.

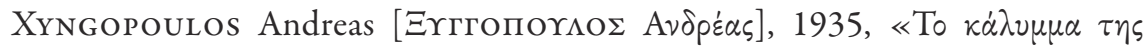

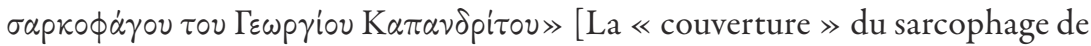

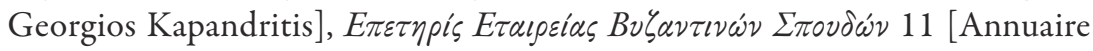
de la société d'études byzantines 11], p. 64-78.

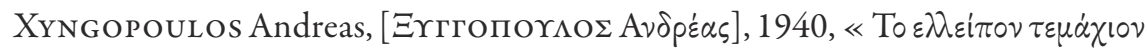

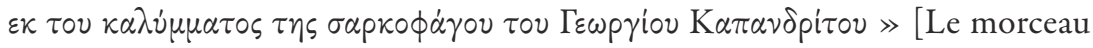
manquant de la «couverture » du sarcophagi de Georgios Kapandritis],

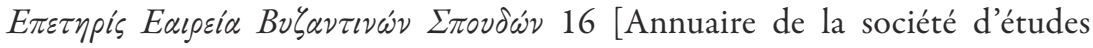
byzantines 16], p. 145-148. 